\title{
Photoplethysmogram Signal Morphology-Based Stress Assessment
}

\author{
Mantas Rinkevičius ${ }^{1}$, Spyridon Kontaxis², Eduardo Gil², Raquel Bailón ${ }^{2}$, Jesús Lázaro ${ }^{2,3}$, \\ Pablo Laguna ${ }^{2}$, Vaidotas Marozas ${ }^{1}$ \\ ${ }^{1}$ Biomedical Engineering Institute and Department of Electronics Engineering, Kaunas University of \\ Technology, Kaunas, Lithuania \\ ${ }^{2}$ Biomedical Signal Interpretation and Computational Simulation (BSICoS) group, Aragón Institute \\ of Engineering Research (I3A), IIS Aragón, University of Zaragoza, Zaragoza, Spain and Biomedical \\ Research Networking Center (CIBER), Zaragoza, Spain \\ ${ }^{3}$ Department of Biomedical Engineering, University of Connecticut, Storrs, CT, United States
}

\begin{abstract}
Stress is a healthy natural response to a perceived or actual threat. However, when stress is persistent, it may decrease work productivity, increase the risk of diseases, and affect the quality of life. Stress is reflected in physiological variables, such as heart rate, blood pressure, and pulse wave velocity among others. A photoplethysmogram (PPG) contains information related to pulse rate and blood pressure. This study analyses parameters derived from PPG signal morphology for mental stress assessment.

A low-complexity algorithm is designed using bandpass filtered higher-order derivatives of the PPG signal for estimation of six morphological parameters: the forward pulse wave amplitude $A_{1}$, the systole and diastole durations $T_{1}$ and $T_{d}$, the time delays of reflected waves $T_{12}$ and $T_{13}$ from the renal and iliac sites in the central arteries, and the pulse duration $T_{p}$. The parameters were investigated on a set of 18 healthy subjects by using a modified Trier Social Stress Test.

The results show that the most sensitive PPG morphology parameters to mental stress are the amplitude of forward wave $A_{1}$, the duration of diastole $T_{d}$, the time delay of the reflected wave $T_{13}$, and the pulse-to-pulse interval $T_{p}$.
\end{abstract}

\section{Introduction}

According to the World Health Organization mental stress has become a global epidemic in the $21^{\text {st }}$ century [1]. Research results show that $25 \%$ of workers have health problems due to work-related mental stress [2]. A low level and short duration stress for a person can be beneficial as it encourages a concentrated, faster and more efficient work. However, a high level of persistent stress may cause physical and psychological health problems, such as impairment in cardiovascular system [3], and kidney disease [4]. Therefore, continuous outpatient monitoring of stress is becoming increasingly relevant [5].

Stress can be indirectly detected by analysis of physiological responses, e.g., it increases heart rate and blood pressure. One way to keep track of heart rate changes is by using electrocardiogram (ECG) registration. Unfortunately, continuous ECG registration is inconvenient for the user. A less obtrusive method to estimate heart rate is photoplethysmography (PPG). The PPG signal can be continuously recorded at a single point on the body, such as at the wrist. Besides heart rate, PPG can be used for estimating blood pressure based on its morphology [6]. Thus, the reliability of the stress detection can be enhanced by combining heart rate and morphological information both extracted from PPG signal.

It has been found [7] that the timing of local minima in the second derivative of hemodynamic signal coincide with the positions of three pulse components - forward and two reflected waves, and the delays of two reflected waves from the renal and iliac sites in the central arteries correlate with arterial blood pressure [8]. In this work, the relationship between human mental stress and PPG morphological parameters extracted from the analysis of bandpass filtered higher-order derivatives of the PPG signal is investigated.

\section{Material and Methods}

\subsection{Data}

A database, composed of biomedical signals recorded from healthy participants who were subjected to mental stress induced by a modified Trier Social Stress Test (TSST) $[9,10]$, was used in this study. A subset of 18 participants was analyzed due to signal quality issues. 
PPG signals were recorded from finger with sampling rate $250 \mathrm{~Hz}$. The stages of protocol and the illustration of raw PPG signal are shown in Figure 1.

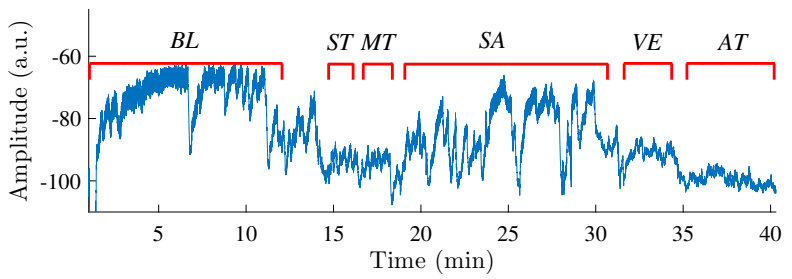

Figure 1. Stages of study protocol and raw PPG signal example: $B L$ - baseline, $S T$ - story telling, $M T$ - memory task, $S A$ - stress anticipation, $V E$ - video exposition, $A T$ - arithmetic task.

The details of the modified TSST protocol are provided below:

1) $B L$ - baseline with relaxation.

2) $S T$ - story telling. Three stories were told to the subject with a great amount of details requiring a lot of attention to remember.

3) $M T$ - memory task. The participant was requested to retell all remembered details about three stories within 30 seconds.

4) $S A$ - stress anticipation. The participant was requested to wait alone for the evaluation of the memory test.

5) $V E$ - video exposition. The presentation of a video with the subject performance in the memory test was shown. A video of an actor remembering all the details was displayed before that, trying to make the subject believe that he/she did not perform the task well enough.

6) $A T$ - arithmetic task. The subject had to perform successive subtractions of 13 , starting from the number 1022 . In case of a calculation error, the subject was requested to restart from 1022 again.

The $B L$ stage was considered as not stressful and was used as a reference for comparison against the last five stages.

\subsection{Preprocessing}

PPG signal processing comprised signal preprocessing, fiducial point detection and estimation of morphological parameters (see Figure 2).

A 4-th order pass-band zero phase Butterworth filter with cut off frequencies 0.4 to $9 \mathrm{~Hz}$ was used to minimize the PPG signal noise and remove slow baseline. The upper cut-off frequency of $9 \mathrm{~Hz}$ ensures a fairly good compromise between high frequency noise rejection and detectability of local maxima/minima points in the PPG and its derivatives. The lower cut-off frequency of $0.4 \mathrm{~Hz}$ is not sufficient to remove baseline wander. Therefore, an

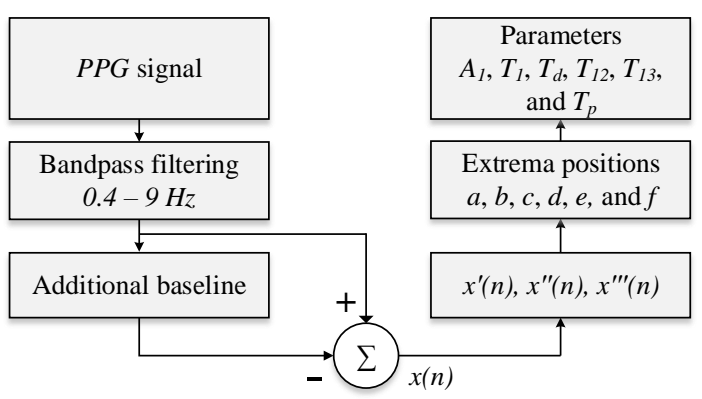

Figure 2. The block diagram of the PPG signal morphological parameters estimation.

additional baseline was estimated using cubic spline interpolation of the PPG pulse feet. This baseline estimate was subsequently subtracted from the passband filtered PPG, ensuring that each pulse starts and ends at zero amplitude value. The foot of each pulse was detected using a slope sum function method proposed in [11].

\subsection{Morphological Parameters}

Morphological parameters (see Figure 3) were selected considering: a) fiducial points, detected by using higherorder derivatives of the PPG signal, indicate the three waves $A_{1}, A_{2}$ and $A_{3}$ (located at $T_{1}, T_{2}$, and $T_{3}$, respectively), of the forward pulse $P_{1}$ and two reflections $P_{2}$ and $P_{3}$ from the central arteries, b) the time delays $T_{12}$ between $P_{1}$ and $P_{2}$, and $T_{13}$ between $P_{1}$ and $P_{3}$ can be considered as surrogates of instantaneous vessel stiffness due to changes of blood pressure [7].

(a)

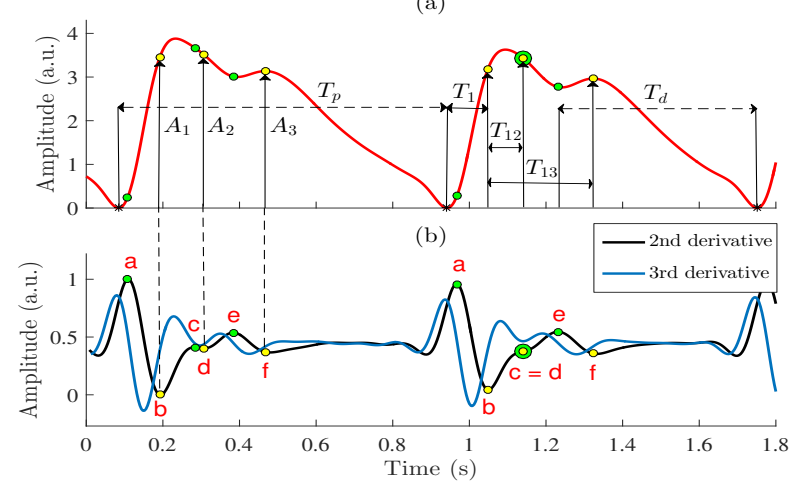

Figure 3. Detection of fiducial points and estimation of morphological parameters: (a) PPG signal, (b) second and third derivatives with green points indicating local maxima of the second derivative, and yellow points - local minima of the second derivative). 
Additional morphological parameters were studied - the diastolic interval $T_{d}$ estimated from the dicrotic notch until the end of the pulse, and the pulse-to-pulse interval $T_{p}$.

The calculation of the first three PPG signal derivatives was required for finding the positions of the extremas ( $a$, $b, c, d, e$ and $f$ ) in the second and third signal derivatives which subsequently were used for estimation of six features (Figure 3). Extremity point locations were detected using the methodology proposed by Charlton [12]. The pulse was defined only if all six extremities were found. Based on these positions, parameters $A_{1}, T_{1}, T_{d}, T_{12}, T_{13}$ and $T_{p}$ were estimated.

The amplitude $A_{1}$ was defined as the value of the PPG signal at the extremity position $b$. The systole duration $T_{1}$ was defined as the time interval from the starting point of the pulse to the $b$ position. The diastole duration $T_{d}$ was defined as the time interval from the $e$ extremity position to the end point of the pulse. The reflection time delays $T_{12}$ and $T_{13}$ were estimated as the time intervals from $b$ to $d$ and from $b$ to $f$ extrema positions, respectively. The sixth morphological parameter, total pulse duration $T_{p}$, was calculated as the time interval from the starting point to the end point of the pulse.

\section{Results}

The nonparametric Wilcoxon rank sum test was used to investigate the significance of the statistical differences between the morphological parameters during basal and stress sessions. Individual parameters were normalized with respect to the mean value of the parameter among all subjects during the BL stage, since the parameters of each participant at rest differ due to the differences in physiology. The difference between baseline $B L$ and stress stages ( $S T, M T, S A, V E, A T$ ) was considered significant when $p<0.05$. The boxplot diagrams of the analyzed parameters are shown in Figure 4.

In addition, the effect size was evaluated for statistical analysis of the parameters in this study (Table 1). The Cohen's d method [13] was used for this purpose.

\section{Discussion}

In this study, the analysis of PPG signal morphological parameters was performed by using the database of 18 participants subjected to the modified Trier Social Stress test. The results (Figure 4 and Table 1) show that the most stress sensitive morphological parameters of PPG signal are the amplitude of the forward wave $A_{1}$, the duration of diastole $T_{d}$, the time delay of the third reflection $T_{13}$, and the pulse-to-pulse interval $T_{p}$.

Mental stress is commonly known to increase sympathetic nerve activity which in turn leads to constriction of peripheral arteries and decrease of blood volume. Thus, an (a) $A_{1}$

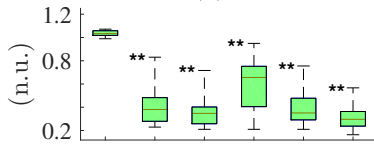

(c) $T_{d}$

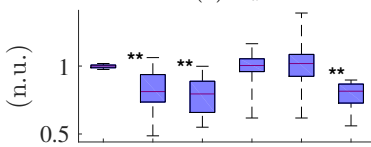

(e) $T_{12}$

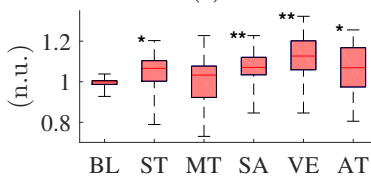

(b) $T_{1}$

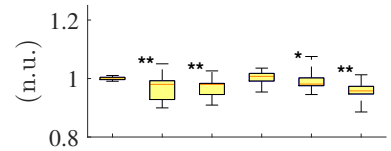

(d) $T_{13}$

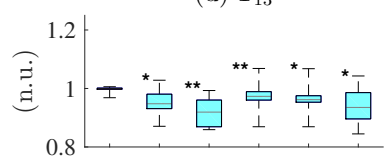

(f) $T_{p}$

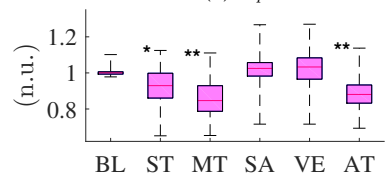

Figure 4. The boxplots of the normalized parameters: (a) amplitude of $P_{1}$, (b) systolic interval $T_{1}$, (c) diastolic interval $T_{d}$, (d) time delay between $P_{1}$ and $P_{3}$, (e) time delay between $P_{1}$ and $P_{2}$, (f) pulse-to-pulse interval $T_{p}$. Significant differences relative to $B L$ are marked with * $(p<0.05)$ and $^{* *}(p<0.001)$.

Table 1. Cohen's $d$ effect sizes. Huge effect size relative to $B L$ is marked with *** $(|d|>2)$, very large effect size is marked with $^{* *}(|d|>1.2)$ and large effect size is marked with * $(|d|>0.8)$.

\begin{tabular}{cccccc}
\hline \hline Par. & $S T$ & $M T$ & $S A$ & $V E$ & $A T$ \\
\hline$A_{1}$ & $5.09^{* * *}$ & $6.86^{* * *}$ & $2.75^{* * * *}$ & $5.48^{* * *}$ & $9.43^{* * *}$ \\
\hline$T_{1}$ & $1.05^{*}$ & $1.34^{* *}$ & -0.18 & 0.40 & $1.87^{* *}$ \\
\hline$T_{12}$ & $-0.90^{*}$ & -0.25 & $-1.01^{*}$ & $-1.55^{* *}$ & -0.88 \\
\hline$T_{13}$ & $1.34^{* *}$ & $2.31^{* * *}$ & $0.81^{*}$ & 0.66 & $1.14^{*}$ \\
\hline$T_{d}$ & $1.62^{* *}$ & $2.32^{* * *}$ & 0.01 & -0.12 & $2.79^{* * *}$ \\
\hline$T_{p}$ & $1.05^{*}$ & $1.59^{* *}$ & -0.22 & -0.28 & $1.64^{* *}$ \\
\hline \hline
\end{tabular}

increased heart rate during mental stress leads to decreased $T_{p}, T_{13}, T_{d}$, and PPG amplitude, measured at finger since less blood volume reaches the extremities.

Our experimental results only partially agree with a numerical modeling study by Charlton et al. [12]. Charlton et al. did not find any relation between PPG signal amplitude and stress. In contrary, our results show that forward wave (systolic) amplitude $A_{1}$ is the most significant stress indicative feature for all stages of the TSST protocol, which is in agreement with other experimental studies, e.g., [14].

Table 1 suggests that parameters derived from timings of fiducial points also indicate mental stress albeit with different sensitivity to protocol stages. The parameter $T_{13}$, which is related to blood pressure, is sensitive to stress as it was expected. However, the effect size of $T_{13}$ is significantly less than that of diastolic duration $T_{d}$ during the arithmetic task $A T$ stage. This might be related to the de- 
tection of the diastolic wave as proposed by [12] in presence of artifacts, where a local maxima is searched in the PPG and not always in the second derivative.

In most stages, the values of the morphological parameters tended to decrease due to the stress-induced sympathetic activation. However, during the stress anticipation stage $(S A)$ the values increased as can be seen in Figure 4. This can be explained by the tendency of participants to relax after completing the $M T$ task. Overall, the patterns of parameter changes during the protocol stages are similar for all parameters except $T_{12}$, which tended to increase instead of decreasing as anticipated. This parameter must be investigated more thoroughly on different databases.

\section{Conclusions}

The most sensitive PPG morphology parameters to mental stress are the amplitude of forward wave $A_{1}$, the duration of diastole $T_{d}$, the time delay of the reflected wave $T_{13}$, and the pulse-to-pulse interval $T_{p}$, therefore, they can be considered for usage in wearable devices for continuous unobtrusive monitoring.

\section{Acknowledgments}

This work has received funding from European Regional Development Fund (project No. 01.2.2-LMTK-718-01-0030) and EU Structural Funds (project No. 09.3.3-LMT-K-712-10-0222) under the grant agreements with the Research Council of Lithuania (LMTLT), research contract SV9-2264, research projects RTI2018097723-B-I00, LMP44-18, T39-17R, and CIBER de Bioingeniería, Biomateriales y Nanomedicina, also Ibercaja-CAI Research Stay Program (IT 16/18). This project has also received funding from the European Union's Framework Programme for Research and Innovation Horizon 2020 (2014-2020) under the Marie Skłodowska-Curie Grant Agreement No. 745755. Also, the authors would like to thank Dr. A. Petrènas for his valuable comments and suggestions on the paper.

\section{References}

[1] Fink G., Stress: concepts, definition and history. Reference Module in Neuroscience and Biobehavioral Psychology (2017), 1-9.

[2] The European Agency for Safety and Health at Work (EUOSHA) and the European Foundation for the Improvement of Living and Working Conditions (Eurofound). Psychosocial risks in Europe: Prevalence and strategies for prevention (2014), 6-7.

[3] Huang C., Webb H. E., Zourdos M. C., Acevedo E. O., Cardiovascular reactivity, stress, and physical activity. Frontiers in Physiology (2013), 4 (314), 1-13.
[4] Bruce M. A., Griffith D. M., Thorpe R. J., Stress and the kidney. Advances in chronic kidney disease (2015), 22 (1), 46-53.

[5] Smets E., De Raedt W., Van Hoof C., Into the wild: the challenges of physiological stress detection in laboratory and ambulatory settings. IEEE Journal of Biomedical and Health Informatics (2019), 2, 463-473.

[6] Martínez G., Howard N., Abbott D., Lim K., Ward R., Can photoplethysmography replace arterial blood pressure in the assessment of blood pressure? Journal of Clinical Medicine (2018), 7 (316), 1-13.

[7] Baruch M. C., Warburton D. E. R., Bredin S. D., Cote A., Gerdt D. W., Adkins C. M., Pulse decomposition analysis of the digital arterial pulse during hemorrhage simulation. Nonlinear Biomedical Physics (2011), 5 (1), 1-15.

[8] Baruch M. C., Kalantari K., Gerdt D. W., Adkins C. M., Validation of the pulse decomposition analysis algorithm using central arterial blood pressure. BioMedical Engineering OnLine (2014), 13 (96), 1-19.

[9] J. Aguilo, P. Ferrer-Salvans, A. Garcia-Rozo, ..., J. M. Garzon-Rey, Project ES3: attempting to quantify and measure the level of stress, Revista de Neurologia (2015), 61 (534), 405-415.

[10] A. Hernando, J. Lázaro, E. Gil, A. Arza, J. M. Garzón, R. López-Antón, C. de la Cámara, P. Laguna, J. Aguiló, R. Bailón (2016). Inclusion of respiratory frequency information in heart rate variability analysis for stress assessment. IEEE Journal of Biomedical and Health Informatics. Vol. 20, n. 4, pp. 1016-1025.

[11] W. Zong, T. Heldt, G. B. Moody and R. G. Mark, An open-source algorithm to detect onset of arterial blood pressure pulses. Computers in Cardiology (2003), Thessaloniki Chalkidiki, Greece, pp. 259-262.

[12] Charlton P. H., Celka P., Farukh B., Alastruey J., Assessing mental stress from the photoplethysmogram: A numerical study. Physiological Measurement (2018), 39 (5), 1-15.

[13] Sawilowsky S., New effect size rules of thumb. Journal of Modern Applied Statistical Methods (2009), 8 (2), 467-474.

[14] Minakuchi E, Ohnishi E, Ohnishi J, Sakamoto S, Hori M, Motomura M, Hoshino J, Murakami K, Kawaguchi T. Evaluation of mental stress by physiological indices derived from finger plethysmography. J Physiol Anthropol (2013) October $12 ; 32: 17$.

Address for correspondence:

Vaidotas Marozas

Biomedical Engineering Institute, K. Baršauskas str. 59, LT-

51423 Kaunas, Lithuania

vaidotas.marozas@ktu.lt 